\title{
Approximate Confidence Limits for the Ratio of Two Binomial Variates with Unequal Sample Sizes
}

\author{
Hokwon $\mathrm{Cho}^{1, a}$ \\ ${ }^{a}$ Department of Mathematical Sciences, University of Nevada, Las Vegas
}

\begin{abstract}
We propose a sequential method to construct approximate confidence limits for the ratio of two independent sequences of binomial variates with unequal sample sizes. Due to the nonexistence of an unbiased estimator for the ratio, we develop the procedure based on a modified maximum likelihood estimator (MLE). We generalize the results of Cho and Govindarajulu (2008) by defining the sample-ratio when sample sizes are not equal. In addition, we investigate the large-sample properties of the proposed estimator and its finite sample behavior through numerical studies, and we make comparisons from the sample information view points.
\end{abstract}

Keywords: Approximate confidence limits, ratio of two binomial proportions, modified MLE, sampleratio, large-sample properties.

\section{Introduction}

The ratio of two binomial proportions and constructing its confidence interval represents an important tool to measure risk ratio (Katz et al., 1978; Bailey, 1987) or relative risk (Gart, 1985; Gart and Nam, 1988) in comparative prospective studies and in biomedical experiments. The ratio or odds ratio of two binomial proportions is also related to vaccine efficacy and attributable risk (Walter, 1976), which arises frequently in epidemiological problems (e.g. cohort study involving two groups).

Among sequential methods for constructing an interval for an unknown parameter based on the fixed-sample size, Ray (1957) and Starr (1966) studied the fixed-width confidence interval for the mean of a normal distribution. Khan (1969) explored a general method to determine stopping rules to obtain a fixed-width confidence interval for an unknown parameter involving some possible unknown nuisance parameters. In addition, Siegmund (1982) investigated a sequential confidence interval for the odds ratio.

The article is organized as follows. In Section 2, we begin with the notations and describe the characteristics of the problem and the proposed method. In Section 3, we study the desirable properties of the proposed estimator in terms of asymptotics. In Section 4, we examine the properties of the proposed procedure. Then, finally in Section 5, we illustrate the procedure with the Monte Carlo examples to make brief comparisons and summarize our conclusions with further remarks.

\section{Formulation}

Suppose that $X_{1}, X_{2}, \ldots$ and $Y_{1}, Y_{2}, \ldots$ are two independent sequences of Bernoulli random variables with probabilities $0<p_{0}<1$ and $0<p_{1}<1$, respectively. Define a ratio, $\theta=p_{1} / p_{0}$. With samples of size $n$ on $X$ and $n_{1}$ on $Y$, let $R=\sum_{i=1}^{n} X_{i}$ and $S=\sum_{j=1}^{n_{1}} Y_{j}$, assuming that $n_{1}=\kappa \cdot n$ such that $\kappa \cdot n$ is

\footnotetext{
${ }^{1}$ Associate Professor, Department of Mathematical Sciences, University of Nevada, Las Vegas, 4505 Maryland Parkway Box 454020, Las Vegas, NV 89154-4020, USA. E-mail: cho@unlv.nevada.edu
} 
an integer and $\kappa$ is known. We call the constant $k\left(=n_{1} / n\right)$ the sample-ratio. We want to find optimal values of $n$ (and $n_{1}$ ) and construct an interval having specified width $2 d$ and confidence coefficient $\gamma$

$$
P\{|\hat{\theta}-\theta| \leq d\} \geq \gamma .
$$

Then, $R$ and $S$ are two independent binomial random variables with parameters $\left(n, p_{0}\right)$ and $\left(n_{1}, p_{1}\right)$, respectively. Since there does not exist an unbiased estimator of the ratio $\theta$, we consider a modified estimator

$$
\hat{\theta}_{n}=\frac{1}{\kappa}\left(\frac{S+1 / 2}{R+1 / 2}\right) .
$$

When we observe $R=r$ and $S=s$, the likelihood (of $\theta$ and $p_{0}$ ) is

$$
L\left(\theta, p_{0}\right)=\left(\begin{array}{l}
n \\
r
\end{array}\right)\left(\begin{array}{c}
n_{1} \\
s
\end{array}\right) p_{0}^{r+s}\left(1-p_{0}\right)^{n-r} \theta^{s}\left(1-p_{0} \theta\right)^{n_{1}-s}
$$

in which $p_{1}=p_{0} \theta$. From the $\log$-likelihood function of $\theta$ and $p_{0}, l\left(\theta, p_{0}\right)$, we have the following maximum likelihood estimates:

$$
\hat{p}_{0, n}=\frac{r}{n}
$$

and since $\hat{\theta}_{m l e}=(1 / \kappa)(S / R)=n S / n_{1} R$, we have

$$
\hat{\theta}_{m l e}=\frac{s}{n_{1} \hat{p}_{0}} .
$$

Furthermore, the Fisher information about $\theta$ is given by

$$
E\left[-\frac{\partial^{2} l\left(\theta, p_{0}\right)}{\partial \theta^{2}}\right]=\frac{n_{1} p_{0}}{\theta\left(1-\theta p_{0}\right)},
$$

and the information about $p_{0}$ is given by

$$
E\left[-\frac{\partial^{2} l\left(\theta, p_{0}\right)}{\partial p_{0}^{2}}\right]=\frac{n}{p_{0}\left(1-p_{0}\right)}+\frac{n_{1} \theta}{p_{0}\left(1-p_{0}\right)} .
$$

Similarly, the joint information about $\theta$ and $p_{0}$ is

$$
E\left[-\frac{\partial^{2} l\left(\theta, p_{0}\right)}{\partial \theta \partial p_{0}}\right]=\frac{n_{1}\left(1-p_{1}\right)}{1-p_{1}}+\frac{n_{1}\left(1-p_{1}\right) p_{1}}{\left(1-p_{1}\right)^{2}}=\frac{n_{1}}{1-\theta p_{0}} .
$$

It follows from Equations (2.4)-(2.6), the information matrix about $\left(\theta, p_{0}\right)$ denoted by $\mathbf{I}\left(\theta, p_{0}\right)$, is then

$$
\mathbf{I}\left(\theta, p_{0}\right)=\left[\begin{array}{cc}
\frac{n_{1} p_{0}}{\theta\left(1-\theta p_{0}\right)} & \frac{n_{1}}{1-\theta p_{0}} \\
\frac{n_{1}}{1-\theta p_{0}} & \frac{n}{p_{0}\left(1-p_{0}\right)}+\frac{n_{1} \theta}{p_{0}\left(1-p_{0}\right)}
\end{array}\right],
$$


and the determinant of the information matrix, $\operatorname{det} \mathbf{I}$ becomes

$$
\operatorname{det} \mathbf{I}=\frac{n_{1} n}{\theta\left(1-p_{0}\right)\left(1-\theta p_{0}\right)} .
$$

Hence, from the inverse of $\mathbf{I}$, namely, $\mathbf{I}^{-1}$ we have the asymptotic variance of $\hat{\theta}_{\text {mle }}$

$$
\begin{aligned}
\operatorname{var}\left(\hat{\theta}_{m l e}\right) & =\frac{\theta\left(1-p_{0}\right)\left(1-\theta p_{0}\right)}{n_{1} n}\left[\frac{n}{p_{0}\left(1-p_{0}\right)}+\frac{n_{1} \theta}{p_{0}\left(1-p_{0}\right)}\right] \\
& =\frac{\theta\left(1-p_{0} \theta\right)}{n_{1} p_{0}}+\frac{\theta^{2}\left(1-p_{0}\right)}{n p_{0}}
\end{aligned}
$$

For the special case $n_{1}=n$, Equation (2.8) reduces to

$$
\operatorname{var}\left(\hat{\theta}_{m l e}\right)=\frac{\theta\left(1-p_{0} \theta\right)+\theta^{2}\left(1-p_{0}\right)}{n p_{0}}=\frac{\theta\left(1+\theta-2 \theta p_{0}\right)}{n p_{0}}
$$

which coincides with the result obtained in Cho and Govindarajulu (2008, Equation (1.10)).

\section{Properties of the Estimator $\hat{\theta}_{\boldsymbol{n}}$}

In this section, we investigate the desirable properties of the modified estimator $\hat{\theta}_{n}$ for the proposed procedure. Even though, there is no unbiased estimator of the true ratio $\theta$, the modified estimator is asymptotically unbiased and so $\hat{\theta}_{m l e}$ is. Therefore, we must show the asymptotic equivalence of the estimators, $\hat{\theta}_{n}$ and $\hat{\theta}_{m l e}$ through their variances.

\subsection{Asymptotic unbiasedness of $\hat{\theta}_{n}$}

Consider the expectation of $\hat{\theta}_{n}$. That is,

$$
E\left(\hat{\theta}_{n}\right)=\frac{1}{\kappa} E\left(\frac{S+1 / 2}{R+1 / 2}\right)=\frac{1}{\kappa} E\left(S+\frac{1}{2}\right) E\left(\frac{1}{R+1 / 2}\right) .
$$

In order to expand, we can rewrite

$$
\begin{aligned}
E\left(\frac{1}{R+1 / 2}\right) & =E\left(\frac{1}{n p_{0}+R-n p_{0}+1 / 2}\right) \\
& =\frac{1}{n p_{0}} E\left[\left(1+\frac{R-n p_{0}+1 / 2}{n p_{0}}\right)^{-1}\right] .
\end{aligned}
$$

Then, after algebraic simplification

$$
\begin{aligned}
E\left(\frac{1}{R+1 / 2}\right) & =\frac{1}{n p_{0}} E\left[1-\left(\frac{R-n p_{0}+1 / 2}{n p_{0}}\right)+\left(\frac{R-n p_{0}+1 / 2}{n p_{0}}\right)^{2}+\cdots\right] \\
& =\frac{1}{n p_{0}}\left[1-\frac{1}{2 n p_{0}}+\frac{n p_{0}\left(1-p_{0}\right)}{\left(n p_{0}\right)^{2}}+\frac{1}{4\left(n p_{0}\right)^{2}}+\cdots\right] .
\end{aligned}
$$


Combining Equations (3.1) and (3.2), we have

$$
\begin{aligned}
E\left(\hat{\theta}_{n}\right)= & \frac{1}{\kappa}\left(n_{1} p_{1}+\frac{1}{2}\right)\left\{\frac{1}{n p_{0}}\left[1-\frac{1}{2 n p_{0}}+\frac{n p_{0}\left(1-p_{0}\right)}{\left(n p_{0}\right)^{2}}+\frac{1}{4\left(n p_{0}\right)^{2}}+\cdots\right]\right\} \\
= & \frac{1}{\kappa}\left\{\frac{n_{1} p_{1}}{n p_{0}}\left[1-\frac{1}{2 n p_{0}}+\frac{n p_{0}\left(1-p_{0}\right)}{\left(n p_{0}\right)^{2}}+\frac{1}{4\left(n p_{0}\right)^{2}}+\cdots\right]\right. \\
& \left.+\frac{1}{2 n p_{0}}\left[1-\frac{1}{2 n p_{0}}+\frac{n p_{0}\left(1-p_{0}\right)}{\left(n p_{0}\right)^{2}}+\frac{1}{4\left(n p_{0}\right)^{2}}+\cdots\right]\right\} \\
= & \frac{1}{\kappa}\left\{\frac{n_{1} p_{1}}{n p_{0}}\left[1-O\left(n^{-2}\right)\right]+\frac{1}{2 n p_{0}}\left[1-O\left(n^{-2}\right)\right]\right\} .
\end{aligned}
$$

Therefore, for sufficiently large $n$

$$
E\left(\hat{\theta}_{n}\right) \simeq \frac{1}{\kappa} \frac{(\kappa n) p_{1}}{n p_{0}}=\frac{p_{1}}{p_{0}}=\theta .
$$

Thus, $\hat{\theta}_{n}$ is an asymptotically unbiased estimator of $\theta$.

Next, we investigate the variance of the modified estimator $\hat{\theta}_{n}$.

\subsection{Asymptotic variance of $\hat{\theta}_{n}$}

Now, we obtain the asymptotic variance of $\hat{\theta}_{n}=\kappa^{-1}(S+1 / 2) /(R+1 / 2)$ assuming that $\kappa n=n_{1}$ is an integer.

\section{Theorem 1.}

$$
\lim _{n \rightarrow \infty} n \operatorname{Var}\left(\hat{\theta}_{n}\right)=\frac{\theta\left(1-p_{0} \theta\right)}{\kappa p_{0}}+\frac{\theta^{2}\left(1-p_{0}\right)}{p_{0}}=\frac{\theta\left(1-p_{0} \theta\right)+\kappa \theta^{2}\left(1-p_{0}\right)}{\kappa p_{0}}
$$

Proof: Noting that

$$
\operatorname{var}\left(\hat{\theta}_{n}\right)=\frac{1}{\kappa^{2}} \operatorname{var}\left(\frac{S+1 / 2}{R+1 / 2}\right)
$$

refer directly to Theorem 1.1 in Cho and Govindarajulu (2008).

\subsection{Asymptotic normality of $\hat{\theta}_{n}$}

From the modified ratio given in Equation (2.2), we have

$$
\begin{aligned}
\sqrt{\kappa n}\left(\frac{S / n_{1}+1 / 2 n_{1}}{R / n+1 / 2 n}-\theta\right) & =\sqrt{\kappa n}\left(\frac{\hat{p}_{1, n}+1 / 2 n_{1}}{\hat{p}_{0, n}+1 / 2 n}-\theta\right) \\
& =\sqrt{\kappa n}\left\{\frac{\hat{p}_{1, n}-p_{1}+p_{1}+1 / 2 n_{1}-\theta\left(\hat{p}_{0, n}+1 / 2 n\right)}{\hat{p}_{0, n}+1 / 2 n}\right\} \\
& =\sqrt{\kappa n}\left\{\frac{\hat{p}_{1, n}-p_{1}+1 / 2 n_{1}-\theta\left(\hat{p}_{0, n}-p_{0}+1 / 2 n\right)}{\hat{p}_{0, n}+1 / 2 n}\right\} \\
& =\sqrt{\kappa n}\left(\frac{\hat{p}_{1, n}-p_{1}}{p_{0}}\right)-\theta \sqrt{\kappa n}\left(\frac{\hat{p}_{0, n}-p_{0}}{p_{0}}\right)+o_{p}(1),
\end{aligned}
$$


where $\hat{p}_{0, n}=r / n$ and $\hat{p}_{1, n}=s / n$. For sufficiently large $n$ and from Slutsky's theorem, the above modification transforms to

$$
\sqrt{\kappa n}\left(\hat{\theta}_{n}-\theta\right) \stackrel{d}{\simeq} N\left\{0, \frac{\theta\left(1-p_{1}\right)}{p_{0}}+\frac{\kappa \theta^{2}\left(1-p_{0}\right)}{p_{0}}\right\} \equiv N\left(0, \sigma^{2}\right),
$$

where

$$
\sigma^{2}=\frac{\theta\left\{1-\theta p_{0}+\theta \kappa\left(1-p_{0}\right)\right\}}{p_{0}}=\frac{\theta\left\{1+\theta \kappa-\theta p_{0}(1+\kappa)\right\}}{p_{0}} .
$$

Now we consider determining $n$ such that

$$
P\{|\hat{\theta}-\theta| \leq d\}=P\left\{\frac{\sqrt{\kappa n}|\hat{\theta}-\theta|}{\sigma} \leq \frac{d \sqrt{\kappa n}}{\sigma}\right\} \geq \gamma .
$$

Thus,

$$
2 \Phi\left(\frac{d \sqrt{\kappa n}}{\sigma}\right)-1 \geq \gamma
$$

or

$$
\frac{d \sqrt{\kappa n}}{\sigma} \geq z_{\frac{(1+\gamma)}{2}}=z \text { (say), }
$$

for specified $d(>0)$ where $\Phi\left(z_{(1+\gamma) / 2}\right)=(1+\gamma) / 2$.

Hence,

$$
n \geq \kappa^{-1}\left(\frac{z \sigma}{d}\right)^{2}
$$

Moreover, the optimal fixed-sample size for the procedure becomes the smallest integer $n^{*}$ such that $n \leq n^{*} \leq n+1$, for estimating $\theta$ with specified $d$ and $z$. That is,

$$
n^{*}=\left\lfloor\kappa^{-1}\left(\frac{z \sigma}{d}\right)^{2}\right\rfloor+1,
$$

where $L \cdot\rfloor$ indicates the greatest integer function.

However, since both $\theta$ and $p_{0}$ are unknown, we resort to the following adaptive sequential rule: We stop sampling after $N$ observations on $X$, and $\kappa N$ observations on $Y$ where

$$
N=\inf _{n}\left\{n \geq m: n \geq \frac{\kappa^{-1} z^{2} \hat{\sigma}_{n}^{2}}{d^{2}}\right\}
$$

where $m(\geq 2)$ is the initial sample size, $\hat{\sigma}_{n}^{2}=\hat{\theta}_{n}\left\{1+\kappa \hat{\theta}_{n}-\hat{\theta}_{n} \hat{p}_{0, n}(1+\kappa)\right\}$ and $\hat{p}_{0, n}=(R+1 / 2) / n$.

Upon stopping we give the $\gamma \times 100 \%$ confidence interval estimate of length $2 d$ for $\theta$ as

$$
\left(\hat{\theta}_{N}-d, \hat{\theta}_{N}+d\right) \text {. }
$$




\section{Asymptotic Properties of the Procedure}

In this section we investigate the asymptotic behavior of the proposed sequential procedure and various properties of the (random) stopping time $N$.

\subsection{Finite sure termination}

Toward its finite sure termination, we have the following theorem:

Theorem 2. Let $N$ be the stopping time associated with the sequential procedure. Then $P\{N<\infty\}$ $=1$.

Proof: Using the stopping rule in Equation (3.5)

$$
\begin{aligned}
P\{N=\infty\} & =\lim _{n \rightarrow \infty} P\{N>n\} \\
& \leq \lim _{n \rightarrow \infty} P\left\{n \leq \kappa^{-1}\left(\frac{z^{2} \hat{\sigma}_{n}^{2}}{d^{2}}\right)\right\}=0
\end{aligned}
$$

since $\hat{\sigma}_{n}^{2}$ converges in probability to $\sigma^{2}$ as $n \rightarrow \infty$. Therefore, the proposed sequential procedure terminates finitely with probability one.

\subsection{First order asymptotics}

We apply the criteria given in Chow and Robbins (1965) to establish the asymptotic efficiency and consistency of the procedure as $d$ tends to zero.

The stopping rule given by Equation (3.5) can be written as

$$
N=\inf _{n}\left\{n \geq m: \frac{n}{\kappa^{-1} \theta(z / d)^{2}} \geq \frac{\hat{\sigma}_{n}^{2}}{\theta}\right\},
$$

where $\hat{\sigma}_{n}^{2}=\hat{\theta}_{n}\left\{1+\kappa \hat{\theta}_{n}-\hat{\theta}_{n} \hat{p}_{0, n}(1+\kappa)\right\} / \hat{p}_{0, n}$.

From this, Equation (4.1) takes the form.

$$
N=N(t)=\min _{n}\left\{n \geq m: Y_{n} \leq \frac{g(n)}{t}\right\},
$$

where

$$
\begin{aligned}
Y_{n} & =\frac{\hat{\theta}_{n}}{\theta}\left\{\frac{1+\kappa \hat{\theta}_{n}-\hat{\theta}_{n} \hat{p}_{0, n}(1+\kappa)}{1+\kappa \theta-\theta p_{0}(1+\kappa)}\right\}\left(\frac{p_{0}}{\hat{p}_{0, n}}\right), \\
g(n) & =n,
\end{aligned}
$$

and

$$
t=\kappa^{-1} \theta\left(\frac{z}{d}\right)^{2} \frac{\left\{1+\kappa \theta-\theta p_{0}(1+\kappa)\right\}}{p_{0}} .
$$

Thus, $\left\{Y_{n}\right\}$ is a sequence of random variables such that $Y_{n}>0$ almost surely (a.s.), $\lim _{n \rightarrow \infty} Y_{n}=1$ a.s. because $\hat{p}_{0, n}$ converges a.s. to $p_{0}$ and $\hat{\theta}_{n} / \theta$ converges a.s. to 1 as $n \rightarrow \infty$. Additionally, we see that $g(n) \rightarrow \infty$ and $g(n) / g(n-1) \rightarrow 1$ as $n \rightarrow \infty$. Since the stopping rule $N$ is well-defined and 
non-decreasing as a function of $t$, we can apply the results of Chow and Robbins (1965) and obtain the first order asymptotics for the proposed sequential procedure.

\section{Theorem 3.}

(i) $\lim _{d \rightarrow 0} N=\infty$ a.s.,

(ii) $\lim _{d \rightarrow 0} N / n^{*}=1$ a.s.,

(iii) $\lim _{d \rightarrow 0} P\left\{\left|\hat{\theta}_{N}-\theta\right| \leq d\right\}=\gamma$.

Proof: For (i) and (ii) proceed as in Cho and Govindarajulu (2008). For the proof of (iii), since $N / n^{*}$ converges in probability to one, $\sqrt{n^{*}}\left(\hat{p}_{0, N}-p_{0}\right)$ is asymptotically normal with mean zero and variance $p_{0}\left(1-p_{0}\right)$. Furthermore since $N / n^{*}$ converges in probability to one, $\left.\sqrt{\kappa n^{*}}\left(\hat{p}_{1, \kappa N}-p_{1}\right)\right)$ is asymptotically normal with mean zero and variance $p_{1}\left(1-p_{1}\right)$. Then, from Anscombe's theorem (1952), it follows that $\hat{p}_{0, N}$ converges in probability to $p_{0}$. Using Slutsky's theorem, we infer that

$$
\sqrt{n^{*}}\left(\hat{\theta}_{N}-\theta\right) \stackrel{d}{\simeq} \sqrt{n^{*}}\left\{\frac{\left(\hat{p}_{1, N}-p_{1}\right)}{p_{0}}-\frac{\theta\left(\hat{p}_{0, N}-p_{0}\right)}{p_{0}}\right\} .
$$

Applying the Anscombe's condition specialized for sums of independent and identically distributed (i.i.d.) random variables on the right-hand side in (4.2), it follows that $\sqrt{n^{*}}\left(\hat{\theta}_{N}-\theta\right)$ is asymptotically $N\left(0, \sigma^{2}\right)$ where $\sigma^{2}=\theta\left\{1+\theta \kappa-\theta p_{0}(1+\kappa)\right\} / p_{0}$. Therefore, we have

$$
P\left\{\left|\hat{\theta}_{N}-\theta\right| \leq d\right\}=P\left\{\frac{\sqrt{n^{*}}\left|\hat{\theta}_{N}-\theta\right|}{\sigma} \leq \frac{d \sqrt{n^{*}}}{\sigma}\right\}=\gamma
$$

as $d \rightarrow 0$, and hence, Theorem 3 is proved.

Next, we assert the asymptotic efficiency of the proposed sequential procedure by proceeding as in Cho and Govindarajulu (2008).

\section{Numerical Studies}

\subsection{Simulation setup}

A Monte Carlo experiment is used to investigate the behavior and performance of the stopping rule in the proposed sequential procedure. The results of the experimentation are summarized in the following tables, which show the values of the parameter $\theta$, namely $\theta=1.0,1.5,2.0$ and 4.0 with selected values of $p_{0}, p_{1}$, and the sample-ratio $\kappa, 0<\kappa \leq 1$. For instance, $\kappa=1$ means that both sample sizes $n$ and $n_{1}$ are taken equally. If $\kappa=0.8$, the sample of $X_{i}$ has taken $25 \%$ more than $Y_{j}$ 's, and if $\kappa=0.5$, the sample size of $X_{i}$ is two times more than $Y_{j}$ 's and so on. Without loss of generality (WLOG) we can assume that $p_{0} \leq p_{1}$ and hence consider only situations in which $\theta \geq 1$ because the roles of $X$ and $Y$ can be interchanged when $\theta \leq 1$. Further since $p_{0} \leq p_{1}$, we expect to sample more from the rare population, WLOG, we can assume that $\kappa \leq 1$ for simulation purposes.

In the table, every value in each row is based on 5,000 independent replications with initial sample size $m=10$ for each experiment. Using the sample ratio $\kappa=n / n_{1}=0.8$ or 0.5 , we present the coverage probability $(\mathrm{CP})$ of the interval $\hat{\theta} \pm d$, and the expected stopping time and optimal sample 
Table 1: $\theta=1.0$ with $p_{0}=0.5$ and $p_{1}=0.5$

\begin{tabular}{|c|c|c|c|c|c|c|c|c|c|c|c|}
\hline \multirow{2}{*}{$\gamma$} & \multirow{2}{*}{$d$} & \multicolumn{4}{|c|}{$\kappa=0.8$} & \multicolumn{4}{|c|}{$\kappa=0.5$} & \multicolumn{2}{|c|}{$y_{j}$} \\
\hline & & $\hat{\theta}$ & $\mathrm{CP}$ & $E(N)$ & $n^{*}$ & $\hat{\theta}$ & $\mathrm{CP}$ & $E(N)$ & $n^{*}$ & $E\left(N_{1}\right)$ & $n_{1}^{*}$ \\
\hline \multirow{3}{*}{.90} & .2 & 1.002 & .890 & 163.08 & 169 & .998 & .921 & 262.96 & 271 & 132.25 & 136 \\
\hline & .3 & .999 & .852 & 68.27 & 76 & .996 & .885 & 111.76 & 121 & 56.66 & 61 \\
\hline & .4 & .998 & .838 & 36.12 & 43 & .993 & .849 & 58.84 & 68 & 30.35 & 34 \\
\hline \multirow{3}{*}{.95} & .3 & 1.000 & .941 & 234.04 & 241 & 1.001 & .968 & 378.94 & 385 & 190.22 & 193 \\
\hline & .4 & 1.002 & .922 & 100.37 & 107 & 999 & .946 & 162.83 & 171 & 82.17 & 86 \\
\hline & .5 & .996 & .879 & 52.69 & 61 & .998 & .915 & 87.14 & 96 & 44.38 & 49 \\
\hline
\end{tabular}

Table 2: $\theta=1.5$ with $p_{0}=0.4$ and $p_{1}=0.6$

\begin{tabular}{|c|c|c|c|c|c|c|c|c|c|c|c|}
\hline \multirow[b]{2}{*}{$\gamma$} & \multirow{2}{*}{$d$} & \multicolumn{4}{|c|}{$\kappa=0.8$} & \multicolumn{4}{|c|}{$\kappa=0.5$} & \multicolumn{2}{|c|}{$y_{j}$} \\
\hline & & $\hat{\theta}$ & $\mathrm{CP}$ & $E(N)$ & $n^{*}$ & $\hat{\theta}$ & $\mathrm{CP}$ & $E(N)$ & $n^{*}$ & $E\left(N_{1}\right)$ & $n_{1}^{*}$ \\
\hline \multirow{3}{*}{.90} & .3 & 1.499 & .882 & 173.54 & 184 & 1.502 & .939 & 283.96 & 294 & 142.74 & 147 \\
\hline & .4 & 1.503 & .832 & 92.43 & 104 & 1.500 & .901 & 154.10 & 166 & 77.83 & 83 \\
\hline & .5 & 1.496 & .804 & 55.24 & 66 & 1.499 & .875 & 94.06 & 106 & 47.91 & 53 \\
\hline \multirow{3}{*}{.95} & .3 & 1.498 & .942 & 250.44 & 260 & 1.503 & .973 & 408.86 & 417 & 205.19 & 208 \\
\hline & .4 & 1.504 & .914 & 136.84 & 147 & 1.504 & .960 & 225.63 & 235 & 113.58 & 117 \\
\hline & .5 & 1.504 & .874 & 83.28 & 94 & 1.503 & .936 & 139.17 & 150 & 70.37 & 75 \\
\hline
\end{tabular}

Table 3: $\theta=2.0$ with $p_{0}=0.3$ and $p_{1}=0.6$

\begin{tabular}{|c|c|c|c|c|c|c|c|c|c|c|c|}
\hline \multirow[b]{2}{*}{$\gamma$} & \multirow{2}{*}{$d$} & \multicolumn{4}{|c|}{$\kappa=0.8$} & \multicolumn{4}{|c|}{$\kappa=0.5$} & \multicolumn{2}{|c|}{$y_{j}$} \\
\hline & & $\hat{\theta}$ & $\mathrm{CP}$ & $E(N)$ & $n^{*}$ & $\hat{\theta}$ & $\mathrm{CP}$ & $E(N)$ & $n^{*}$ & $E\left(N_{1}\right)$ & $n_{1}^{*}$ \\
\hline \multirow{3}{*}{.90} & .4 & 1.999 & .875 & 238.63 & 254 & 1.998 & .937 & 391.29 & 406 & 196.41 & 203 \\
\hline & .5 & 1.994 & .825 & 144.17 & 162 & 2.000 & .915 & 243.56 & 260 & 122.56 & 131 \\
\hline & .6 & 2.000 & .787 & 95.88 & 113 & 1.998 & .879 & 162.17 & 181 & 81.93 & 90 \\
\hline \multirow{3}{*}{.95} & .4 & 2.001 & .937 & 347.48 & 361 & 2.004 & .975 & 566.44 & 578 & 283.98 & 289 \\
\hline & .5 & 2.000 & .907 & 214.58 & 231 & 2.001 & .958 & 354.56 & 370 & 178.05 & 185 \\
\hline & .6 & 1.994 & .874 & 141.71 & 160 & 1.997 & .942 & 239.21 & 256 & 120.38 & 128 \\
\hline
\end{tabular}

Table 4: $\theta=4.0$ with $p_{0}=0.2$ and $p_{1}=0.8$

\begin{tabular}{|c|c|c|c|c|c|c|c|c|c|c|c|}
\hline \multirow{2}{*}{$\gamma$} & \multirow{2}{*}{$d$} & \multicolumn{4}{|c|}{$\kappa=0.8$} & \multicolumn{4}{|c|}{$\kappa=0.5$} & \multicolumn{2}{|c|}{$y_{j}$} \\
\hline & & $\hat{\theta}$ & $\mathrm{CP}$ & $E(N)$ & $n^{*}$ & $\hat{\theta}$ & $\mathrm{CP}$ & $E(N)$ & $n^{*}$ & $E\left(N_{1}\right)$ & $n_{1}^{*}$ \\
\hline \multirow{3}{*}{.90} & .6 & 3.995 & .899 & 613.60 & 638 & 3.998 & .961 & 1000.16 & 1022 & 500.84 & 511 \\
\hline & .7 & 4.010 & .874 & 446.07 & 471 & 3.996 & .951 & 726.09 & 751 & 363.82 & 376 \\
\hline & .8 & 3.987 & .856 & 326.70 & 359 & 4.010 & .940 & 553.74 & 577 & 277.65 & 288 \\
\hline \multirow{3}{*}{.95} & .6 & 3.995 & .953 & 884.72 & 906 & 4.000 & .988 & 1433.33 & 1452 & 717.42 & 726 \\
\hline & .7 & 4.008 & .946 & 650.37 & 668 & 4.002 & .984 & 1047.57 & 1067 & 524.54 & 533 \\
\hline & .8 & 3.999 & .922 & 483.61 & 511 & 3.999 & .979 & 793.87 & 816 & 397.69 & 408 \\
\hline
\end{tabular}

size denoted by $E(N)$ and $n^{*}$ for $X_{i}$ 's, and $E\left(N_{1}\right)$ and $n_{1}^{*}$ for $Y_{j}$ 's, respectively. The nominal level of confidence $\gamma$ for the interval is .90 or .95 for each value of $\theta$.

From Table 1 to Table 4, we observe that the expected stopping time $E(N)$ monotonically increases (to infinity) as the sample ratio $\kappa$ becomes smaller (i.e., sample of $x_{i}$ 's getting more) or $d$ decreases (to zero). We observe that as $d$ decreases the coverage probability (CP) is getting close (eventually) to the nominal probability $\gamma$, which is referred to as asymptotic consistency. It should be noted that if one takes $X_{i}$ 's (or $Y_{j}$ 's) more, then the CP is comparatively higher than the one in equal sample sizes. Therefore, the above numerical evidence indicates that the finite-sample behavior lends support to the asymptotic behavior of the proposed sequential procedure when $d \rightarrow 0$.

Increasing the starting sample size $m$ results in the increase of both $E(N)$ and CP. Accordingly, when the $\mathrm{CP}$ is below the nominal level, choosing a moderate size of $m$ is a trade-off for obtaining a 
Table 5: $\theta=2.0$ with $p_{0}=0.3$ and $p_{1}=0.6$

\begin{tabular}{|c|c|c|c|c|c|c|}
\hline Sample-Ratio & $d=0.4$ & $\gamma=0.90$ & \multicolumn{2}{|c|}{$X_{i} \sim \operatorname{Ber}\left(p_{0}\right)$} & \multicolumn{2}{|c|}{$Y_{j} \sim \operatorname{Ber}\left(p_{1}\right)$} \\
\hline$\kappa=n_{1}^{*} / n^{*}$ & $\hat{\theta} \pm d$ & $\mathrm{CP}$ & $E(N)$ & $n^{*}$ & $E\left(N_{1}\right)$ & $n_{1}^{*}$ \\
\hline (1) $K=1$ & $(1.599,2.399)$ & 0.841 & 196.41 & 203 & 196.41 & 203 \\
\hline (2) $\kappa=0.8$ & $(1.599,2.399)$ & 0.875 & 238.63 & 254 & 196.41 & 203 \\
\hline (3) $K=0.5$ & $(1.598,2.398)$ & 0.937 & 391.29 & 406 & 196.41 & 203 \\
\hline
\end{tabular}

Table 6: $\theta=4.0$ with $p_{0}=0.2$ and $p_{1}=0.8$

\begin{tabular}{|c|c|c|c|c|c|c|}
\hline Sample-Ratio & $d=0.7$ & $\gamma=0.95$ & \multicolumn{2}{|c|}{$X_{i} \sim \operatorname{Ber}\left(p_{1}\right)$} & \multicolumn{2}{|c|}{$Y_{j} \sim \operatorname{Ber}\left(p_{0}\right)$} \\
\hline$\kappa=n_{1}^{*} / n^{*}$ & $\hat{\theta} \pm d$ & $\mathrm{CP}$ & $E(N)$ & $n^{*}$ & $E\left(N_{1}\right)$ & $n_{1}^{*}$ \\
\hline (1) $k=1$ & $(3.305,4.705)$ & 0.902 & 524.54 & 533 & 524.54 & 533 \\
\hline (2) $\kappa=0.8$ & $(3.308,4.708)$ & 0.946 & 650.37 & 668 & 524.54 & 533 \\
\hline (3) $k=0.5$ & $(3.302,4.702)$ & 0.984 & 1047.57 & 1067 & 524.54 & 533 \\
\hline
\end{tabular}

higher coverage probability. For practical purposes, the size of $d$ can be determined from the standard error (S.E.) of the estimate $\hat{\theta}$.

\subsection{Comparison: Equal-sample sizes versus Unequal-sample sizes}

In this subsection we compare the results from the unequal-sample sizes with values of the sampleratio $\kappa=0.8$ and $\kappa=0.5$ with the results from the equal-sample sizes $(\kappa=1)$ on $x$ and $y$. For brevity, we summarize and present part of the results in the following two tables, Tables 5-6 for $\theta=2.0$ with $90 \%$ nominal level and $\theta=4.0$ with $95 \%$ nominal level, respectively. Each table shows three values of $\kappa$, the coverage probability (CP), and the expected stopping times $E(N)$ for each values of $\kappa$.

From the above tables, as the sample-ratio $\kappa$ decreases (i.e., take more samples with smaller $p$ ) we observe that the coverage probability $(\mathrm{CP})$ for the interval of width $2 d$ improves. For instance, when sample-ratio $\kappa=0.8$, comparing to the equal sample sizes $(\kappa=1)$, the CPs have increased by $3.4 \%$ points for $\gamma=0.90$ and $4.4 \%$ points for $\gamma=0.95$, respectively. Therefore, for a more stable estimation of the ratio for two binomial variates it is reasonable to take more samples from the population having smaller probability $p$ even though the equal-sample sizes minimize the expected stopping times..

The expected stopping time $E(N)$ is uniformly bigger than $E\left(N_{1}\right)$ when $\kappa<1$. At the same time, it seems to be generally true that $\kappa<1$ gives the higher CP than the one with equal-sample sizes; however, we need to note that eventually the $\mathrm{CP}$ approaches the nominal level $\gamma$ as $d$ gets smaller. From these, we surmise that reducing variability (in the denominator of the statistic used) by taking (reasonably) more samples (i.e., getting 'more' information from the rare population) seems to be fair and is a better idea for a more stable estimation of the true ratio $\theta$.

\subsection{Concluding remarks}

We have proposed a sequential method to obtain the approximate confidence limits for the ratio of two binomial variates that may have unequal sample sizes. The proposed method offers a relatively new perspective on the information aspects. The procedure is developed based on a modified MLE in terms of the sample-ratio $\kappa$; subsequently, the large-sample properties of the proposed estimator are investigated. The finite sample behavior was verified through numerical studies. In addition, by comparing the expected stopping times and the coverage probabilities of the intervals, it is recommended to take more samples from the rare population to have more precise intervals for the ratio parameter $\theta=p_{1} / p_{0}$.

We also note that it might be preferred to have a confidence interval based on the likelihood-ratio since it would be invariant. Future studies should include dealing with advantages and disadvantages 
in practical usages between two approaches even though they are not completely comparable.

\section{Acknowledgements}

The author wishes to thank the referee for the insightful review of this manuscript which resulted in a more accurate and readable paper.

\section{References}

Anscombe, F. J. (1952). Large sample theory of sequential estimation, Proceedings of the Cambridge Philosophical Society, 48, 600-607.

Bailey, B. J. R. (1987). Confidence limits to the risk ratio, Biometrics, 43, 201-205.

Cho, H. (2007). Sequential risk-efficient estimation for the ratio of two binomial proportions, Journal of Statistical Planning and Inference, 137, 2336-2346.

Cho, H. and Govindarajulu, Z. (2008). Sequential confidence limits for the ratio of two binomial proportions, International Journal of Statistics and Management System, 3, 26-42.

Chow, Y. S. and Robbins, H. (1965). On the asymptotic theory of fixed-width sequential confidence intervals for the mean, The Annals of Mathematical Statistics, 36, 457-462.

Gart, J. (1985). Approximate tests and interval estimation of the common relative risk in the combination of $2 \times 2$ tables, Biometrika, 72, 673-677.

Gart, J. and Nam, J. (1988) Approximate interval estimation of the ratio of binomial parameters: A review and corrections for skewness, Biometrics, 44, 323-338.

Katz, D., Baptista, J., Azen, S. and Pike, M. (1978). Obtaining confidence intervals for the risk ratio in cohort studies, Biometrics, 34, 469-474.

Khan, R. (1969). A general method of determining fixed-width confidence intervals, The Annals of Mathematical Statistics, 40, 704-709.

Ray, D. (1957). Sequential confidence intervals for the mean of a normal distribution with unknown variance, Journal of the Royal Statistical Society, Series B, 19, 133-143.

Siegmund, D. (1982). A sequential confidence interval for the odds ratio, Probability and Mathematical Statistics, 2, 149-156.

Starr, N. (1966). The performance of a sequential procedure for the fixed-width interval estimation of the mean, The Annals of Mathematical Statistics, 37, 36-50.

Walter, S. (1976). The estimation and interpretation of attributable risk in health research, Biometrics, 32, 829-849. 